\title{
Prognostic value of the autophagy markers LC3 and p62/ SQSTM1 in early-stage non-small cell lung cancer
}

\author{
Anna M. Schläfli ${ }^{1}$, Olivia Adams ${ }^{1,2}$, José A. Galván ${ }^{1}$, Mathias Gugger ${ }^{1,3}$, Spasenija \\ Savic $^{4}$, Lukas Bubendorf ${ }^{4}$, Ralph A. Schmid ${ }^{5}$, Karl-Friedrich Becker ${ }^{6}$, Mario P. \\ Tschan $^{1,2}$, Rupert Langer ${ }^{1}$ and Sabina Berezowska ${ }^{1}$ \\ ${ }^{1}$ Institute of Pathology, University of Bern, Bern, Switzerland \\ ${ }^{2}$ Graduate School for Cellular and Biomedical Sciences, University of Bern, Bern, Switzerland \\ 3 Promed Laboratoire Médical, Marly, Switzerland \\ ${ }^{4}$ Institute of Pathology, Universty Hospital Basel, Basel, Switzerland \\ ${ }^{5}$ Division of General Thoracic Surgery, Inselspital University Hospital Bern, Bern, Switzerland \\ ${ }^{6}$ Institute of Pathology, Technische Universität München, München, Germany \\ Correspondence to: Sabina Berezowska, email: sabina.berezowska@pathology.unibe.ch
}

Keywords: autophagy, LC3, p62/SQSTM1, immunohistochemistry, non-small cell lung cancer

Received: April 09, $2016 \quad$ Accepted: May 19, $2016 \quad$ Published: May 26, 2016

\section{ABSTRACT}

Autophagy is a cellular degrading process that promotes tumor cell survival or cell death in cancer, depending on the progress of oncogenesis. Protein light chain 3 (LC3) and p62/SQSTM1 (p62) are associated with autophagosomal membranes that engulf cytoplasmic content for subsequent degradation. We studied LC3 and p62 expression using immunohistochemistry in a large cohort of 466 stage I/II non-small cell lung cancer (NSCLC) using a tissue microarray. We evaluated dot-like cytoplasmic expression of LC3 and dot-like, cytoplasmic and nuclear staining for p62 in relation to clinico-pathological parameters.

LC3 expression correlated with all p62 patterns, as those correlated among each other ( $p<0.001$ each). There was no correlation with stage, age or gender. A combination of high LC3/high p62 dot-like staining (suggesting impaired autophagy) showed a trend for better outcome $(p=0.11)$. Interestingly, a combined low cytoplasmic/low nuclear p62 expression regardless of dot-like staining was an independent prognostic factor for longer survival $(p=0.006 ; H R=1.96)$, in addition to tumor stage $(p=0.004 ; \mathrm{HR}=1.4)$.

The autophagy markers LC3 and p62 are differentially expressed in NSCLC, pointing towards a biologically significant role. High LC3 levels seem to be linked to lower tumor aggressiveness, while high general p62 expression was significantly associated with aggressive tumor behavior.

\section{INTRODUCTION}

Lung cancer is one of the most frequent cancers, and responsible for the most deaths due to malignant disease. The desire for better understanding its biology, which could ideally confer additional, better and more precise oncological therapeutics, is thus enormous. Nonsmall cell lung cancer (NSCLC) comprises the majority of lung carcinomas. Most of NSCLC can be histologically typed as adenocarcinoma (AC), squamous cell carcinoma (SqCC) or large cell carcinoma (LCC), as defined by the WHO [1].

The cellular process of autophagy has many functions including bulk degradation of cytoplasmic content to ensure homeostasis [2], degradation of superfluous or damaged organelles [3] and the engulfment 
of pathogens [4]. The role of autophagy in cancer is complex and depends on tumor stage, type and the driving oncogene [5-7]. Under homeostatic conditions autophagy is regarded as a tumor suppressor process, but there is evidence it is later on required for tumor progression $[8$, 9].

Exploiting autophagy for anti-cancer therapeutic benefit is an area gaining ever increasing focus. On a cellular level autophagy aims at degrading cytoplasmic content in the lysosomal compartment [2]. Macroautophagy, referred to as autophagy from here on, is hallmarked by the formation of double-membrane vesicles, so-called autophagosomes, which finally fuse with lysosomes to degrade their content [10].

On a molecular level several functional complexes, comprising so-called autophagy related ( $A T G)$ genes, are involved in autophagosome biogenesis, cargo recognition and in mediating autophagosome-lysosome fusion. Briefly, the unc-51-like kinase 1 (ULK1) complex is important for autophagy initiation. Down-stream of the ULK-1 complex the coiled-coil myosin-like BCL2-interacting protein (Beclin1) complex is generating phosphoinositol3-phosphates (PI3Ps), allowing for the recruitment of other ATG proteins [11]. Furthermore, two ubiquitin-like systems are required for vesicle elongation and cargo recognition, namely the ATG12-ATG5- and the ATG8conjugation system. The latter one serves to lipidate ATG8 family members, including microtubule-associated protein 1 light chain 3 (MAP1LC3 or LC3, hereafter referred to as LC3) $[12,13]$. LC3 comprises three isoforms LC3A, B and C. Since LC3 is incorporated into the inner and outer membrane of autophagosomes [14], its detection allows for monitoring the autophagy pathway [15]. LC3-I is cytosolic, whereas the lipidated form LC3-II is membrane bound [14].

Autophagy can either be non-selective or can very specifically target certain portions of the cytoplasm for degradation [16]. Different autophagy receptors are mediating selectivity by being able to interact with the cargo on the one hand and the autophagy machinery on the other hand [17]. The best studied is sequestosome 1 (SQSTM1 or p62, hereafter referred to as p62). Essential for its function as an autophagy receptor is its ability to interact with ubiquitinated cargo and LC3B [18]. During this process p62 itself is constantly degraded. Reduced levels of p62 are therefore associated with an activated autophagy pathway [15]

Both LC3 and p62 are frequently used as markers to assess autophagy [15]. Although autophagy is a flux, and should ideally be measured in functional assays, immunohistochemistry is the method of choice for tissue based retrospective analysis of large cohorts. Thereby, dot-like staining of LC3 serves as a surrogate marker for autophagic vesicles. As p62 is constantly degraded in autolysosomes, it is a surrogate marker for autophagic degradation.
In NSCLC, there are only very few studies on the prognostic significance of the expression of autophagy markers, and all have been conducted in broad stage collectives $[19,20]$. Because the role of autophagy in cancer may be stage dependent, the aim of our present study was to assess autophagy-associated markers in nonmetastasized, early-stage NSCLC.

\section{RESULTS}

\section{Staining patterns}

Formalin-fixed and paraffin embedded (FFPE) tissue of 466 primary resected, chemotherapy-naïve, early-stage NSCLC was analyzed for the expression of autophagy associated markers LC3 and p62. The anti-LC3 antibody from Novus $(\mathrm{N})$ is known to recognize the $\mathrm{LC} 3$ isoforms A and B [21] the anti-LC3B antibody from Cell Signaling (CS) is isoform B specific [22].

Staining of both antibodies could be assessed in 442 cases, and correlated significantly $(p<0.0001)$, although the CS antibody showed a generally weaker staining, with few positive cases. LC3 (CS) dot-like staining could be evaluated in 464 cases: score 0 in 403 cases $(86.9 \%)$, score 1 in 47 cases $(10.1 \%)$, score 2 in 11 cases $(2.4 \%)$, score 3 in 3 cases $(0.6 \%)$.

LC3 (N) dot-like staining could be evaluated in 443 cases and was observed as score 0 in 240 cases $(54.2 \%)$, score 1 in 120 cases $(27.1 \%)$, score 2 in 48 cases $(10.8 \%)$ and score 3 in 35 cases $(7.9 \%)$ (Figure 1A). Stone-like structures (SLS)[20] were present in 8 cases $(1.8 \%)$. For p62 dot-like staining, 420 punches were suitable for evaluation, and 328 cases $(78.1 \%)$ scored 0,59 cases (14\%) scored 1, 24 cases (5.7\%) scored 2 and 9 cases $(2.1 \%)$ scored 3. SLS were present in 14 cases $(3.3 \%)$. Cytoplasmic staining of p62 was absent (score 0) in 82 cases $(19.5 \%)$, score 1 in 266 cases $(63.3 \%)$ and score 2 in 72 cases $(17.1 \%)$. Nuclear staining was absent in 264 cases $(62.9 \%)$ and present in 156 cases $(37.1 \%)$.

LC3 dot-like staining showed a significant correlation with all p62 staining patterns ( $p<0.001$ each), and all p62 staining patterns correlated among each other ( $p<0.001$ each). Examples for p62 cytoplasmic, dot-like, nuclear and SLS staining are shown in Figure 1B.

Assessment of staining heterogeneity was performed in 38 exemplary cases, using 8 punches from two tissue blocks of each tumor. Completely homogenous staining with regard to score 1 to 3 was observed in 12/38 cases (31.6\%) for LC3 dot-like staining, 13/38 cases (34.2\%) for p62 dot-like staining, 18/38 cases (47.4\%) for p62 cytoplasmic staining and $19 / 38$ cases $(50 \%)$ for p62 nuclear staining. Heterogeneous staining, however, with a deviation of $>1$ scoring points in $>1$ spot, that would also have caused a different classification into „low“ and 

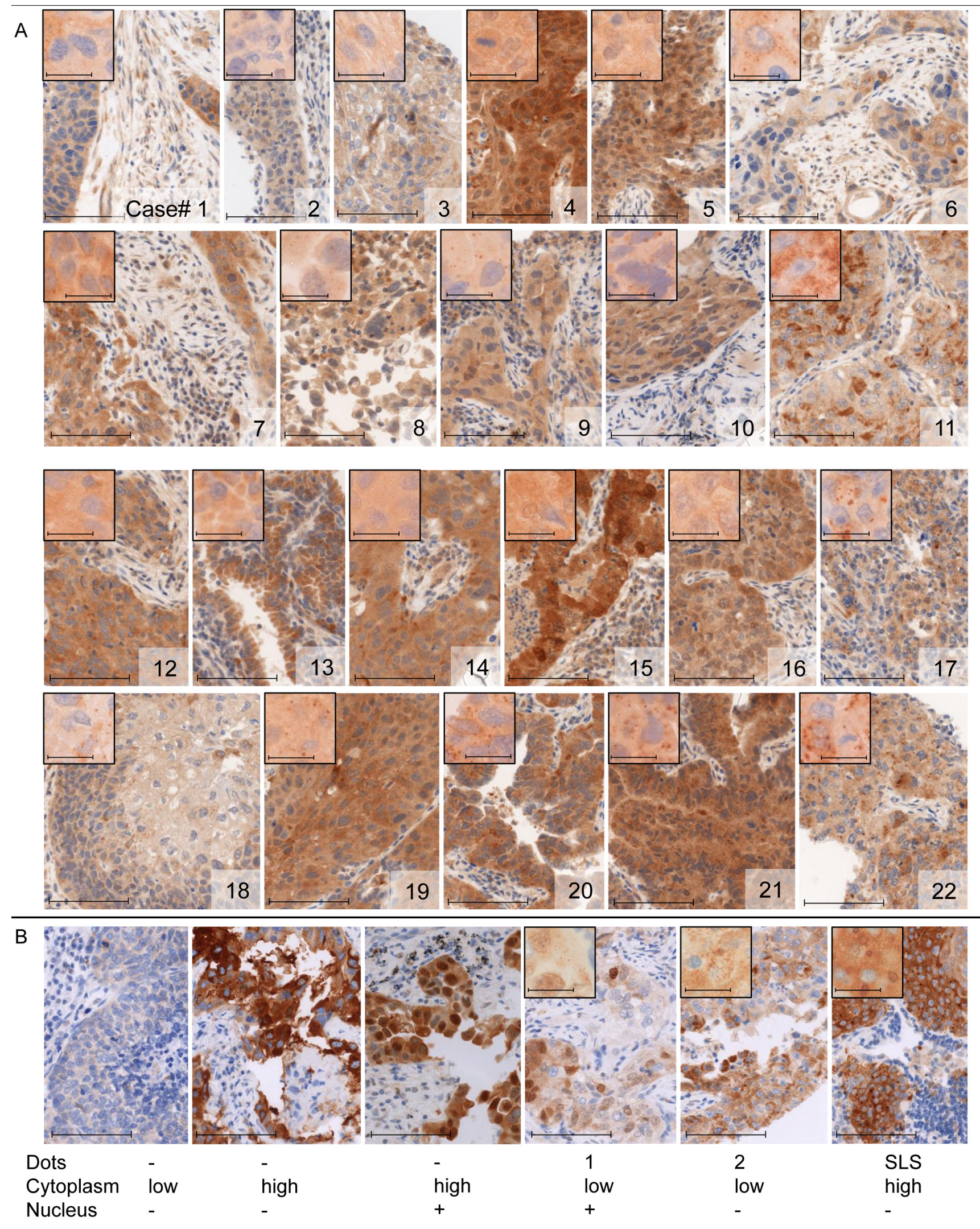

Figure 1: Immunohistochemical staining of LC3 and p62. A. LC3 (Novus) staining patterns of the 22 cases further analysed by immunoblot analysis (figure 2). Results of dot-like staining patterns are given in the upper panels of figure $2 \mathrm{~A}$ and $\mathrm{B}$ for each case. B. Examples of p62 staining, with cytoplasmic, dot-like, nuclear and SLS staining patterns. (original magnification $400 \mathrm{x}$, scale bar $100 \mu \mathrm{m}$; insets: original magnification $1000 \mathrm{x}$, scale bar $20 \mu \mathrm{m}$ ). 
Table 1: LC3 (Novus) and p62 dot-like staining and clinico-pathologic parameters (total $n=466$; for evaluation LC3 $n=443 ; \mathrm{p} 62 n=420$ ).

\begin{tabular}{|c|c|c|c|c|c|c|c|c|}
\hline \multirow[t]{2}{*}{ parameter } & & \multirow{2}{*}{\begin{tabular}{|l} 
total \\
$\mathbf{N}(\%)$
\end{tabular}} & \multicolumn{3}{|c|}{ LC3 dot-like staining } & \multicolumn{3}{|c|}{ p62 dot-like staining } \\
\hline & & & low & high & $p$ & low & high & $p$ \\
\hline total & & \begin{tabular}{|l|}
466 \\
$(100)$ \\
\end{tabular} & 360 & 83 & & 328 & 92 & \\
\hline \multicolumn{9}{|l|}{ age } \\
\hline & $<67$ years & $247(53)$ & 191 & 47 & \multirow{2}{*}{0.556} & 169 & 53 & \multirow{2}{*}{0.302} \\
\hline & $\geq 67$ years & $219(47)$ & 169 & 36 & & 159 & 39 & \\
\hline \multicolumn{9}{|l|}{ gender } \\
\hline & male & \begin{tabular}{|l|}
343 \\
$(73.6)$
\end{tabular} & 276 & 53 & \multirow{2}{*}{0.016} & 237 & 68 & \multirow{2}{*}{0.753} \\
\hline & female & \begin{tabular}{|l|}
123 \\
$(26.4)$ \\
\end{tabular} & 84 & 30 & & 91 & 24 & \\
\hline \multicolumn{9}{|l|}{ histology } \\
\hline & $A d C A$ & $\begin{array}{l}202 \\
(43.3)\end{array}$ & 146 & 48 & \multirow{3}{*}{0.017} & 144 & 38 & \multirow{3}{*}{0.463} \\
\hline & $S q C C$ & \begin{tabular}{|l|}
229 \\
$(49.1)$ \\
\end{tabular} & 186 & 30 & & 164 & 45 & \\
\hline & $L C C$ & $35(7.5)$ & 28 & 5 & & 20 & 9 & \\
\hline \multicolumn{9}{|l|}{ pT category } \\
\hline & $1 a$ & $24(5.2)$ & 17 & 4 & \multirow{5}{*}{0.789} & 14 & 6 & \multirow{5}{*}{0.108} \\
\hline & $1 b$ & $31(6.7)$ & 23 & 4 & & 13 & 9 & \\
\hline & $2 a$ & $\begin{array}{l}304 \\
(65.2) \\
\end{array}$ & 233 & 59 & & 217 & 61 & \\
\hline & $2 b$ & $68(14.6)$ & 56 & 9 & & 52 & 9 & \\
\hline & 3 & $39(8.4)$ & 31 & 7 & & 32 & 7 & \\
\hline \multicolumn{9}{|l|}{ UICC stage } \\
\hline & $I A$ & $55(11.8)$ & 40 & 8 & \multirow{4}{*}{0.666} & 27 & 15 & \multirow{4}{*}{0.076} \\
\hline & $I B$ & $\begin{array}{l}304 \\
(65.2) \\
\end{array}$ & 233 & 59 & & 217 & 61 & \\
\hline & IIA & $68(14.6)$ & 56 & 9 & & 52 & 9 & \\
\hline & IIB & $39(8.4)$ & 31 & 7 & & 32 & 7 & \\
\hline
\end{tabular}

„high“" as described below (refer to following paragraph Correlation with clinico-pathologic parameters) was observed in only $2 / 38$ cases $(5.3 \%)$ for LC3 dot-like staining, $1 / 38$ cases $(2.6 \%)$ for p62 dot-like staining, no case for p62 cytoplasmic staining and $1 / 38$ cases $(2.6 \%)$ for p62 nuclear staining.

\section{Immunoblot analysis}

During the process of autophagosome formation LC3 gets cleaved and lipidated before incorporation into the membrane. This modification enables to distinguish between cytosolic LC3 (LC3-I) and membrane-bound LC3 (LC3-II) on a western blot [23]. Immunoblot analysis of 22 cases selected according to absent and strongly present LC3 dot-like staining revealed the feasibility of this methodology for LC3 evaluation in FFPE tissue. The results correlated mostly with the immunohistochemical staining patterns as depicted in Figure 1 versus 2. Importantly, both LC3 antibodies (from Novus and Cell Signaling) showed equal results on Western Blot (Figure 2).

\section{Correlation with clinico-pathologic parameters}

For the purposes of correlation with pathological and clinical parameters, immunohistochemistry scores were categorized as either "low" or "high" for each staining pattern according to our established protocol [24] with slight modifications, following the prognostic impact of the single scores: For LC3 dot-like staining scores 0 and 1 were classified as low, scores 2 and 3 as high. For p62 dot-like staining score 0 was classified as low, scores 1,2 and 3 were interpreted as high. For p62 cytoplasmic 
A

\begin{tabular}{|l|c|c|c|c|c|c|c|c|c|c|c|}
\hline $\begin{array}{l}\text { Case } \\
\text { Number }\end{array}$ & $\mathbf{1}$ & $\mathbf{2}$ & $\mathbf{3}$ & $\mathbf{4}$ & $\mathbf{5}$ & $\mathbf{6}$ & $\mathbf{7}$ & $\mathbf{8}$ & $\mathbf{9}$ & $\mathbf{1 0}$ & $\mathbf{1 1}$ \\
\hline $\begin{array}{l}\text { LC3 Novus } \\
\text { IHC Score }\end{array}$ & 0 & 0 & 0 & 0 & 0 & 2 & 2 & 2 & 3 & 3 & 3 \\
\hline $\begin{array}{l}\text { LC3B CS } \\
\text { IHC Score }\end{array}$ & 0 & 0 & 0 & 0 & 0 & 1 & 1 & 2 & 2 & 2 & 3 \\
\hline
\end{tabular}
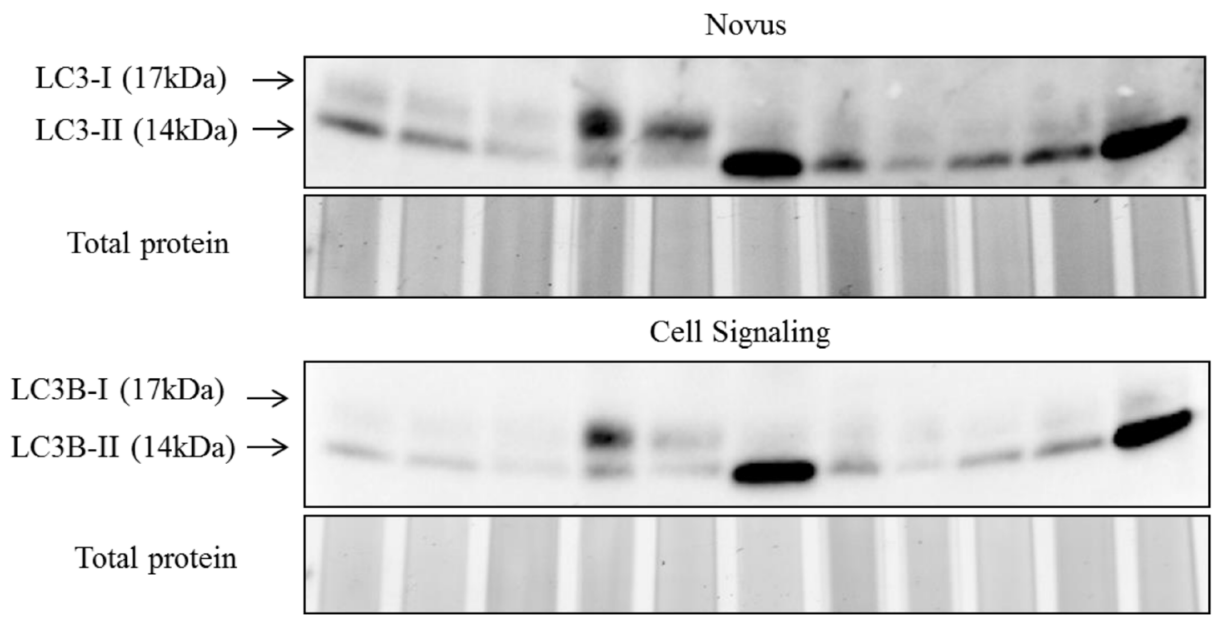

B

\begin{tabular}{|l|c|c|c|c|c|c|c|c|c|c|c|}
\hline $\begin{array}{l}\text { Case } \\
\text { Number }\end{array}$ & 12 & 13 & 14 & 15 & 16 & 17 & 18 & 19 & 20 & 21 & 22 \\
\hline $\begin{array}{l}\text { LC3 Novus } \\
\text { IHC Score }\end{array}$ & 0 & 0 & 0 & 0 & 0 & 2 & 2 & 2 & 3 & 3 & 3 \\
\hline $\begin{array}{l}\text { LC3B CS } \\
\text { IHC Score }\end{array}$ & 0 & 0 & 0 & 0 & 0 & 1 & 1 & 2 & 2 & 2 & 3 \\
\hline
\end{tabular}

Novus

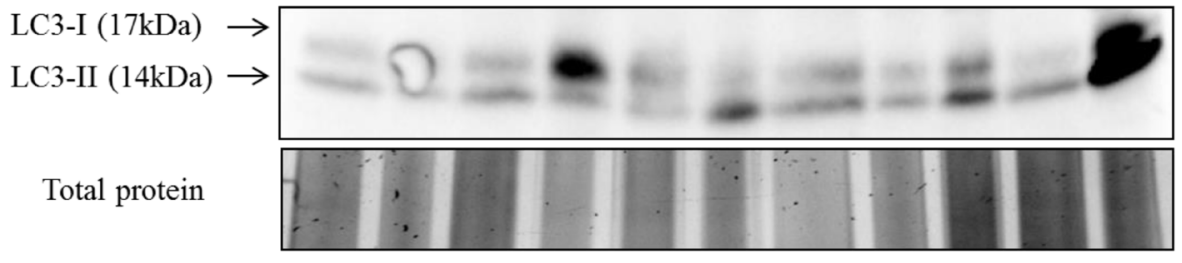

Cell Signaling

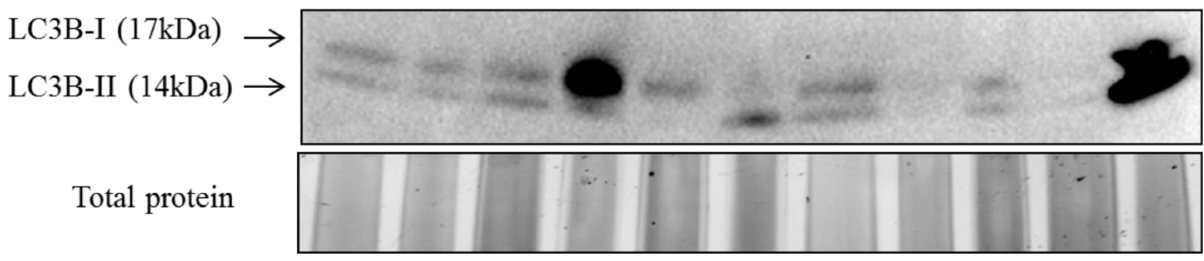

Figure 2: Immunoblot analysis of LC3 in protein extracts from FFPE tissue from 22 early-stage non-small cell lung carcinomas. A. Immunoblot analysis of LC3-I (cytosolic) and LC3-II (membrane-bound) of cases 1 to 12, and B. cases 13 to 22, using anti-LC3 Novus (middle panel) and anti-LC3B Cell Signaling (bottom panel) primary antibodies. Total protein was visualised and used as loading control. Immunohistochemistry scores for anti-LC3 Novus and anti-LC3B Cell Signaling primary antibodies are shown in the tables (upper panel). 
Table 2: p62 cytoplasmic and nuclear staining and clinico-pathologic parameters (total $n=466$; for evaluation $n=420$ ).

\begin{tabular}{|c|c|c|c|c|c|c|c|c|}
\hline \multirow[t]{2}{*}{ parameter } & & \multirow{2}{*}{$\frac{\text { total }}{N(\%)}$} & \multicolumn{3}{|c|}{ p62 cytoplasmic staining } & \multicolumn{3}{|c|}{ p62 nuclear staining } \\
\hline & & & low & high & $p$ & absent & present & $p$ \\
\hline total & & $\begin{array}{l}466 \\
(100)\end{array}$ & 348 & 72 & & 264 & 156 & \\
\hline \multicolumn{9}{|l|}{ age } \\
\hline & $<67$ years & $247(53)$ & 187 & 35 & \multirow{2}{*}{0.428} & 143 & 79 & \multirow{2}{*}{0.442} \\
\hline & $\geq 67$ years & $219(47)$ & 161 & 37 & & 121 & 77 & \\
\hline \multicolumn{9}{|l|}{ gender } \\
\hline & male & $\begin{array}{l}343 \\
(73.6)\end{array}$ & 247 & 58 & \multirow{2}{*}{0.097} & 185 & 120 & \multirow{2}{*}{0.140} \\
\hline & female & $\begin{array}{l}123 \\
(26.4)\end{array}$ & 101 & 14 & & 79 & 36 & \\
\hline \multicolumn{9}{|l|}{ histology } \\
\hline & $A d C A$ & $\begin{array}{l}202 \\
(43.3)\end{array}$ & 161 & 21 & \multirow{3}{*}{0.029} & 134 & 48 & \multirow{3}{*}{$<0.001$} \\
\hline & $S q C C$ & $\begin{array}{l}229 \\
(49.1)\end{array}$ & 164 & 45 & & 112 & 97 & \\
\hline & $L C C$ & $35(7.5)$ & 23 & 6 & & 18 & 11 & \\
\hline \multicolumn{9}{|l|}{ pT category } \\
\hline & $1 a$ & $24(5.2)$ & 17 & 3 & \multirow{5}{*}{0.113} & 13 & 7 & \multirow{5}{*}{0.942} \\
\hline & $1 b$ & $31(6.7)$ & 15 & 7 & & 15 & 7 & \\
\hline & $2 a$ & $\begin{array}{l}304 \\
(65.2) \\
\end{array}$ & 233 & 45 & & 173 & 105 & \\
\hline & $2 b$ & $\begin{array}{l}68 \\
(14.6)\end{array}$ & 47 & 14 & & 38 & 23 & \\
\hline & 3 & $39(8.4)$ & 36 & 3 & & 25 & 14 & \\
\hline \multicolumn{9}{|l|}{ UICC stage } \\
\hline & $I A$ & $\begin{array}{l}55 \\
(11.8)\end{array}$ & 32 & 10 & \multirow{4}{*}{0.145} & 28 & 14 & \multirow{4}{*}{0.894} \\
\hline & $I B$ & $\begin{array}{l}304 \\
(65.2)\end{array}$ & 233 & 45 & & 173 & 105 & \\
\hline & $I I A$ & $\begin{array}{l}68 \\
(14.6)\end{array}$ & 47 & 14 & & 38 & 23 & \\
\hline & $I I B$ & $39(8.4)$ & 36 & 3 & & 25 & 14 & \\
\hline
\end{tabular}

staining scores 0 and 1 were classified as low and score 2 as high. Only 14 cases (3\%) showed high dot-like staining using LC3 (CS), 83 cases (18.7\%) using LC3 (N).

Low LC3 $(\mathrm{N})$ dot-like staining was more frequent in males $(p=0.016)$ and $\operatorname{SqCC}(p=0.017)$. There was no association with age (median), pT category or stage (Table 1). There was no significant association with the abovementioned factors for $\mathrm{p} 62$ dot-like staining or the presence of LC3 positive or $\mathrm{p} 62$ positive SLS. In contrast, lower p62 cytoplasmic and nuclear stainings were more frequent in $\mathrm{AC}(p=0.029$ and $p<0.001$, respectively; Table 2).

\section{Survival analysis}

Survival data was available for 349 patients. Survival analysis showed a better overall survival (OS) and recurrence free survival (RFS) for younger patients (cut-off median; OS $p=0.002$; RFS $p=0.012$ ), for females (OS $p=0.06$; RFS $p=0.035)$, for patients with $\mathrm{AC}$ and LCC (OS $p=0.005$; RFS $p=0.025)$, and with lower $\mathrm{pT}$ categories $/ \mathrm{UICC}$ stages $(\mathrm{OS} p=0.004 / p=0.003$; RFS $p=0.018 / p=0.137$; respectively).

None of the patients with high LC3 (CS) dot-like staining relapsed or died, but short follow up times in this sub-group preclude any conclusions and further analyses.

For LC3 $(\mathrm{N})$, high dot-like staining patterns were in trend linked to a better OS $(p=0.16)$, similar to high p62 dot-like staining $(p=0.28)$, but not to RFS $(p=0.49$; 
Table 3: Multivariate analysis for tumor related overall survival.

\begin{tabular}{|l|l|l|l|l|}
\hline \multirow{2}{*}{ parameter } & \multirow{2}{*}{ HR } & \multicolumn{2}{|l|}{ (5\% confidential interval } & \multirow{2}{*}{$\boldsymbol{p}$-value } \\
\cline { 3 - 4 } & & $\min$ & $\max$ & \\
\hline Age (median) & 1.574 & 0.982 & 2.525 & 0.06 \\
\hline Gender & 0.72 & 0.39 & 1.33 & 0.294 \\
\hline Histology & 1.148 & 0.765 & 1.723 & 0.504 \\
\hline $\boldsymbol{p T}$ category & 1.407 & 1.117 & 1.772 & 0.004 \\
\hline $\boldsymbol{p 6 2}$ cytoplasmic/nuclear & 1.962 & 1.217 & 3.164 & 0.006 \\
\hline
\end{tabular}

$p=0.855)$. The presence of SLS was not associated with survival. In contrast, low p62 cytoplasmic staining was significantly associated with a better tumor related OS ( $p$ $=0.036$ ), similar to negative $\mathrm{p} 62$ nuclear staining (trend; $p=0.066)$ (Figure 3), but not with RFS ( $p=0.091 ; p=$ 0.536 , respectively).

According to previously published work [25] a combination of LC3 and p62 dot-like staining patterns was analyzed: A small subgroup of tumors with both high LC3 and p62 dot-like staining $(n=31)$ was associated with a better OS. In contrast, high LC3/low p62 dot-like pattern $(n=18)$ and low LC3/any level of p62 dot-like pattern $(n$ $=300)$ had a similarly unfavorable prognostic impact ( $p=$ $0.11)$ (Figure 3). This trend was not demonstrated for RFS $(p=0.514)$.

For p62 cytoplasmic and nuclear staining, subgroups were built according to a different recently published paper [26]. Tumors with both p62 low cytoplasmic and nuclear staining $(n=185)$ were associated with a significantly better OS and RFS than mixed $(n=139)$ and both high cytoplasmic and nuclear stained tumors $(n=25)$ (OS $p=$ $0.005 ;$ RFS $p=0.008$ )

In multivariate analysis including UICC pT category/UICC stage, histological subtype, age, gender and p62 cytoplasmic/nuclear staining, only pT category/ UICC stage and low p62 cytoplasmic/nuclear staining were independent prognostic factors for $\mathrm{OS}(\mathrm{HR}=1.96$; 95\%CI 1.2-3.2; $p=0.006$ )(Table 3$)$ and RFS (HR $=1.655$; $95 \% \mathrm{CI} ; 1.1-2.4 ; p=0.011)$.

\section{DISCUSSION}

We investigated a homogeneous, large early-stage NSCLC cohort of 466 patients for the expression of the autophagy markers LC3 and p62, using a previously validated immunohistochemical protocol [24]. The observed differential expression of both markers points towards a biologically significant role in NSCLC, although drawing conclusions from the staining patterns on specific disruptions of the autophagy mechanism in the tumors is not clear-cut.

A trend for better tumor related OS was seen in tumors expressing high dot-like staining of both LC3 and p62, which in a simplified model could imply impairment at late stages of the autophagy cascade. The low number of tumors expressing high LC3 in our cohort may have precluded statistical significance although the results may be valid and important. Corroborating studies are therefore justified. Contrary to the reported adverse prognostic significance of SLS in the SqCC subgroup of NSCLC, using antibodies to the LC3 isoform LC3A [20], detection of SLS was rare and not associated with outcome in our cohort.

p62 serves as a link between LC3B and ubiquitinated substrates to be degraded in autolysosomes, rendering it a surrogate marker for degradation [15]. Thus, high LC3/ low p62 dot-like pattern may be indicative of activated and intact autophagy, and low LC3/p62 any dot-like pattern may show low basal autophagy [25]. Those staining patterns had similarly unfavorable prognostic impact in our collective. However, it is questionable if drawing conclusions from steady-state levels of autophagy markers on the functional state of autophagic activity is a valid approach. As very recently updated, the methodology to assess this highly dynamic process on human FFPE tissue still needs significant improvement [15]. LC3 puncta may mean activated autophagy, but also impaired autophagy due to a late stage block. Second, all autophagy markers may be associated with non-autophagic structures.

Interestingly though, it was high p62 cytoplasmic/ nuclear expression that emerged as an independent factor for shorter tumor related OS and RFS, regardless of histological subtype. This is in line with Inoue et al., who reported high cytoplasmic expression of p62 to be an independent marker for worse prognosis among $\mathrm{AC}$ $(n=72)$, in a cohort of 109 NSCLC, without specifying for nuclear positivity [19]. Similar correlations of high cytoplasmic p62 expression and adverse clinical features were also found in other cancer types, such as breast cancer [27, 28], prostate cancer [29] and oral SqCC [25]. It is important to note, that this cytoplasmic and nuclear expression of p62 may not necessarily be linked to autophagy. One alternative candidate effector may be the NRF2-KEAP1-pathway. Several groups could show that high levels of p62 lead to Nrf2 stabilization and subsequent transcription of genes with an antioxidant function [30]. Importantly, persistent activation of Nrf2 via $\mathrm{p} 62$ stabilization contributes to tumor progression [31]. However, in the study by Inoue et al. accumulation of p62 
A

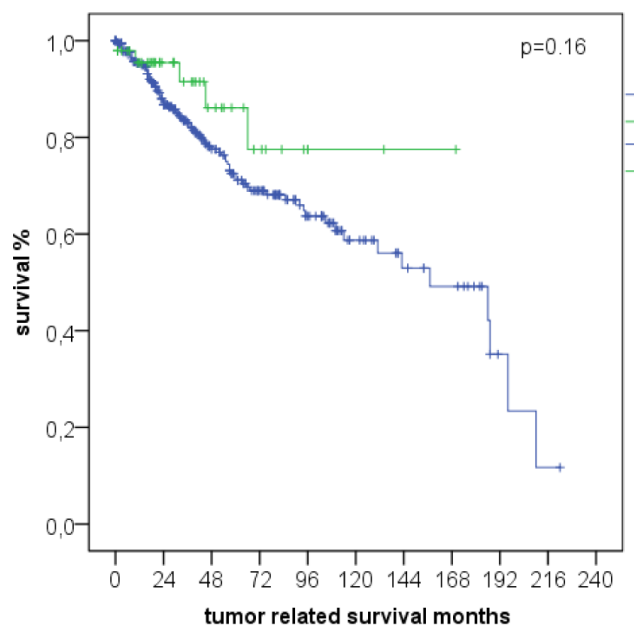

C

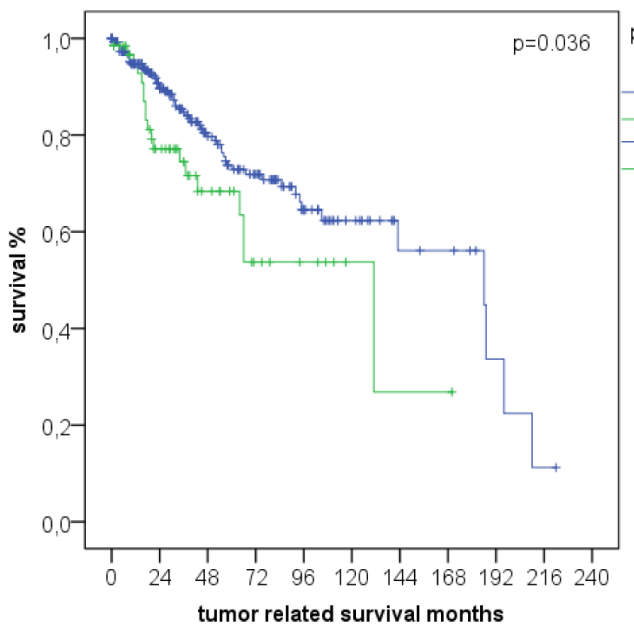

E

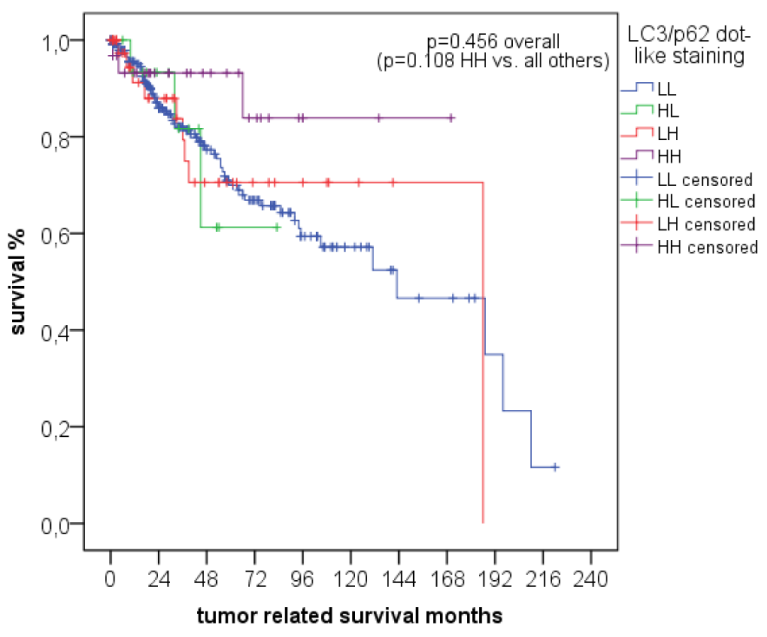

B
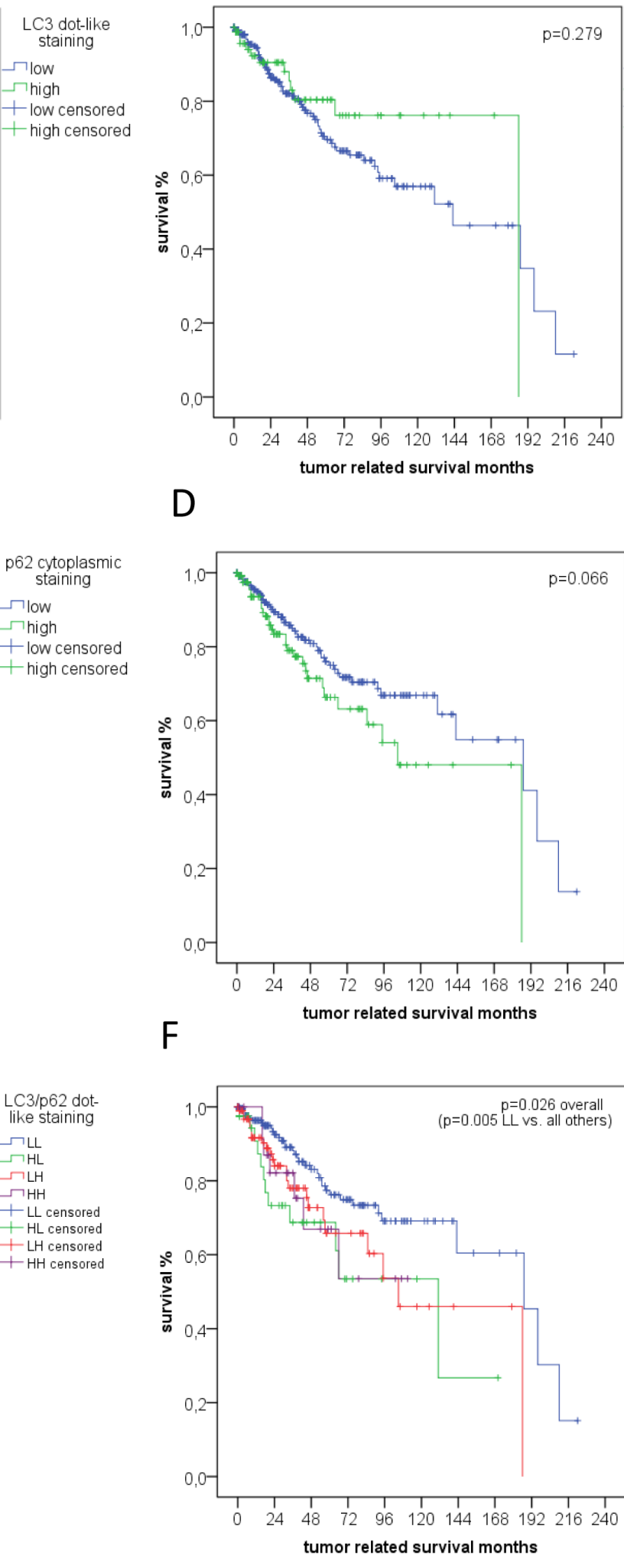

F
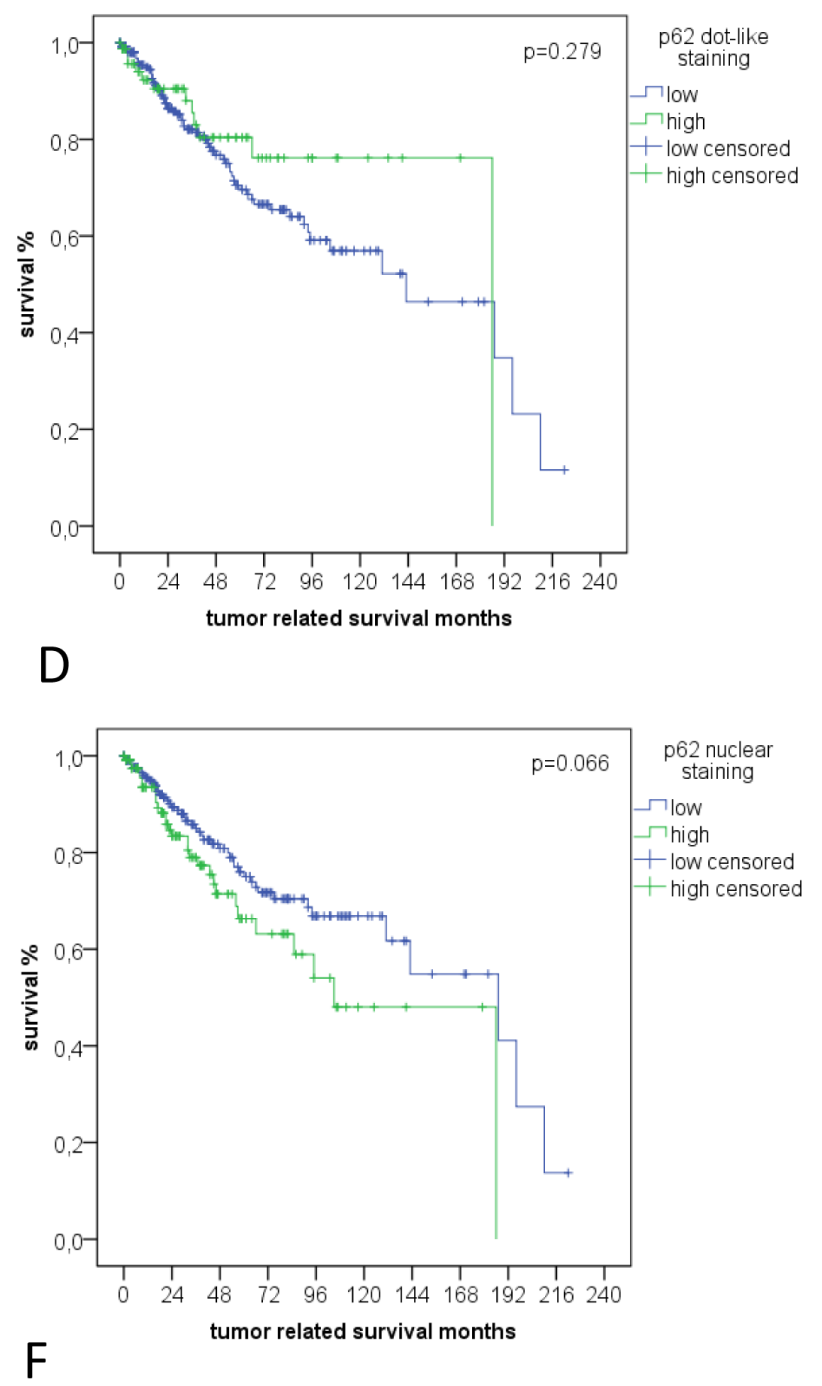

D nuc staining

$-\neg \mathrm{LL}$

$\neg \mathrm{HL}$
$-\neg \mathrm{LH}$
$-\neg \mathrm{HH}$

- LL censored

- HL censored + LH censored - HH censored

Figure 3: Survival analysis. Kaplan Meier curves for tumour related overall survival assessed for A. LC3 and B. p62 dot-like staining, C. cytoplasmic and D. nuclear p62 staining, E. a combination of LC3/p62 dot-like staining (HH = both high, LL = both low, HL = high LC3/low p62, LH = low LC3/high p62), and $\mathbf{F}$. a combination of p62 cytoplasmic and nuclear staining $(\mathrm{HH}=$ both high, $\mathrm{LL}=$ both low, $\mathrm{HL}$ $=$ high cytoplasmic, low nuclear, $\mathrm{LH}=$ low cytoplasmic, high nuclear). 
did not necessarily result in the stabilization of NRF2 [19]. Because increased p62 was shown to lead to NFкBactivation $[32,33]$, another explanation for worse outcome may be the potentiation of NFKB-dependent transcription via p62 [19].

Whereas the cytoplasmic function of p62 is well studied its nuclear function remains less clear. It has been shown that p62 is a protein able to rapidly shuttle between nucleus and cytoplasm, although its preferential localization under homeostatic conditions is cytoplasmic. In the nucleus p62 strongly co-localizes with PML-bodies and is probably involved in delivering ubiquitinated proteins for degradation [34]. Furthermore, p62 is involved in recruiting ALFY, a crucial factor for autophagic degradation of protein aggregates, from the nucleus to the cytoplasm [35]. It might well be that p62 is involved in recruiting other proteins to the cytoplasm as well.

It remains to be investigated whether accumulation of p62 in the nucleus is cancer cell-specific, and if so, what functional consequences it may have. Mislocalization of proteins in cancer cells is seen quite often. The best known is probably the predominant nuclear localization of $\mathrm{NF} \kappa \mathrm{B}$ in many cancer types [36].

In summary, this is the largest study to date reporting the expression of autophagy related markers LC3 and p62 in a well-defined, early-stage NSCLC cohort of 466 cases. We report the correlation of dot-like immunohistochemical staining for LC3 with LC3-II protein expression assessed by immunoblot analysis of the same FFPE archival cases, corroborating the feasibility to assess autophagy structures using immunohistochemistry.

We observed a trend for better outcome in tumors with high dot-like staining of LC3 and p62, being surrogates for autophagic vacuoles and thus the process of autophagy, although low numbers of this subgroup might have precluded statistical significance. Multivariate analysis rendered cytoplasmic/nuclear p62 staining an independent predictor of worse outcome, regardless of LC3 expression.

Our results warrant further investigations concerning the link between expression data and functional autophagy states and a possible non-autophagy related role of p62 in NSCLC.

\section{MATERIALS AND METHODS}

\section{Patient cohort}

The retrospective study included patients with primary resected node-negative early-stage NSCLC (UICC $7^{\text {th }}$ edition 2009 stage IA-IIB)[37], treated with curative surgery and diagnosed at the Institute of Pathology, University of Bern, Switzerland and the Institute of Pathology, University Hospital Basel, Switzerland between January 1988 and August 2008. The detailed staging work-up for this cohort of 544 patients has already been reported [38]. After exclusion of cases with lymph node metastases, neoadjuvant treatment, rare tumor types other than $\mathrm{AC}, \mathrm{SqCC}$ and $\mathrm{LCC}$, and insufficient tumor tissue for further analysis, 466 patients were finally included. This retrospective study was approved by the local ethics committee.

Median age of the patient cohort was 67 years (range; 39-83 years). The detailed distribution of clinicpathologic characteristics can be seen in Tables 1 and 2. The median RFS was 110 months (95\% CI; 85-134 months). Tumor related OS, which was calculated from the day of surgical resection until last contact or tumor specific death, was 186 months (95\% CI; 119-252 months).

\section{Tissue microarray}

A tissue microarray (TMA) was constructed as reported elsewhere [38]. In short, formalin fixed paraffin embedded (FFPE) tissue blocks were retrieved from the archives of the Institutes of Pathology. One punch (diameter $0.6 \mathrm{~mm}$ ) from the tumor center of the bestpreserved block was transferred to the receptor TMAblock.

To assess for staining heterogeneity, 38 patients with positive and negative LC3 staining patterns were selected from the Bern sub-cohort. A TMA was constructed as reported elsewhere [39], using 8 cores (diameter $0.6 \mathrm{~mm}$ ) from 2 FFPE blocks per tumor. Additional punches (4 x 1 $\mathrm{mm}$ per tumor) were taken for protein extraction.

\section{Immunohistochemistry}

Immunohistochemical staining was performed using the automated system BOND RX (Leica Biosystems, Newcastle, UK). TMA sections were cut at $4 \mu \mathrm{m}$, deparaffinized and rehydrated in dewax solution (Leica Biosystems). Endogenous peroxidase activity was blocked with $\mathrm{H}_{2} \mathrm{O}_{2}$ solution for 4 minutes.

All samples were incubated with the following primary antibodies for 30 minutes at room temperature (RT), as described before [24]: LC3B from Cell Signaling Technology (MA, USA, \#3868, clone D11, dilution 1:3000), LC3 from Novus Biologicals (Cambridge, UK, \#NB600-1384, dilution 1:3000) using Tris buffer (pH 9) at $95^{\circ} \mathrm{C}$ for 30 minutes for antigen retrieval, p62 from MBL (IL, USA, \#PM045, dilution 1:8000) using Citrate buffer $(\mathrm{pH} 6.5)$ at $100^{\circ} \mathrm{C}$ for 30 minutes for antigen retrieval. Antibody detection was done with the Bond Polymer Refine Detection kit (Leica Biosystems, DS9800) following the manufacturer's instructions.

Read out of stainings was performed by AMS and 
SB as established before [24], and discrepancies were discussed on a multi-header microscope to gain a final consensus.

Dot-like staining patterns for LC3 and p62 were scored as: 0 - no dots or barely visible dots in $<5 \%$ of cells, 1 - detectable dots in 5-25\% of cells, 2 readily detectable dots in $25-75 \%$ of cells and 3 - dots in $>75 \%$ of cells. Stone like structures (SLS) [20] were recorded separately.

Cytoplasmic p62 staining was scored as: 0 - no or very faint staining, 1 - weak staining, 2 - moderate to strong staining. Nuclear p62 staining was recorded as present or absent.

Images were acquired using a Zeiss Axioplan 2 microscope (objective magnification $40 \mathrm{x}$ and $100 \mathrm{x}$ ) and Axiovision software.

\section{Immunoblot analysis}

Protein was extracted from 4 x $1 \mathrm{~mm}$ tumor punches using the Qproteome FFPE Tissue Kit (Qiagen, 37623) as per manufacturer's instruction. The samples were deparaffinized and rehydrated using Xylene followed by descending alcohol series. $100 \mu \mathrm{l}$ of extraction buffer, containing $\beta$-mercaptoethanol, was added to each sample followed by ice ( 15 minutes) and heat treatment $\left(100^{\circ} \mathrm{C}\right.$, 20 minutes; $80^{\circ} \mathrm{C}, 2$ hours).

Samples were centrifuged and supernatant transferred to a new tube. Protein concentration was determined using the Bradford protein assay (BioRad, Cressier, Switzerland). $30 \mu \mathrm{g}$ of total protein were denatured in $5 \mathrm{x}$ self-made sample buffer, containing $\beta$-mercaptoethanol (Sigma Aldrich, M-7522, Leiden, Netherlands), at $95^{\circ} \mathrm{C}$ for 5 minutes. Samples were loaded and separated on a $4-20 \%$ stain-free pre-cast gel (BioRad). Total protein was visualized as loading control. Separated protein was transferred onto a polyvinylidene difluoride membrane using the Trans-Blot ${ }^{\circledR}$ Turbo $^{\text {TM }}$ Transfer system (BioRad). Membranes were blocked in 5\% bovine serum albumin (BSA)/tris-buffered saline (TBS) for 1 hour at RT. The same primary antibodies were used as for immunohistochemistry in a working concentration of $1: 1000$ in $5 \%$ milk/TBS with $0.1 \%$ Tween (Sigma Aldrich, P9416). Membranes were incubated with primary antibodies over night at $4^{\circ} \mathrm{C}$ with shaking. Subsequently, membranes were incubated with anti-rabbit IgG, HRPlinked Antibody (Cell Signaling, 7074, dilution 1:10 000 in 5\% milk/TBS-T) for 3 hours at RT with shaking. Prior to visualization, membranes were incubated with Clarity Western ECL Substrate (BioRad) for 5 minutes at RT with shaking. Results were visualized using the ChemiDoc ${ }^{\mathrm{TM}}$ MP system (BioRad).

\section{Statistical analysis}

Descriptive and comparative statistical analyses were performed using the SPSS 23 software (SPSS Inc, Chicago, IL, USA). Associations between staining patterns and clinico-pathologic parameters were evaluated using simple cross tabs ( $\mathrm{Chi}^{2}$-test or Fisher's exact test). Survival analysis was performed using log rank test and Cox regression analysis. The significance level was set at 0.05 .

\section{ACKNOWLEDGMENTS}

The authors gratefully acknowledge the Translational Research Unit of the Institute of Pathology, University of Bern, for excellent technical support.

\section{CONFLICTS OF INTEREST}

The authors declare that there is no conflict of interest.

\section{GRANT SUPPORT}

The work was supported by grants from Bernische Krebsliga (to SB), Swiss Cancer League (to RL, KLS3083-02-2013) and Stiftung für Klinisch-Experimentelle Tumorforschung Bern (to MPT).

\section{Editorial note}

This paper has been accepted based in part on peerreview conducted by another journal and the authors' response and revisions as well as expedited peer-review in Oncotarget.

\section{REFERENCES}

1. Travis WD, Brambilla E, Burke AP, Marx A and Nicholson AG. (2015). WHO Classification of Tumours of the Lung, Pleura, Thymus and Heart. In: Bosman FT, Jaffe ES, Lakhani SR and Ohgaki H, eds. World Health Organization Classification of Tumours. (Lyon: International Agency for Research on Cancer (IARC)).

2. Kuma A and Mizushima N. Physiological role of autophagy as an intracellular recycling system: with an emphasis on nutrient metabolism. Semin Cell Dev Biol. 2010; 21:683690.

3. Feng Y, He D, Yao Z and Klionsky DJ. The machinery of macroautophagy. Cell Res. 2014; 24:24-41.

4. Deretic V. Autophagy in immunity and cell-autonomous defense against intracellular microbes. Immunol Rev. 2011; 240:92-104. 
5. White E. The role for autophagy in cancer. J Clin Invest. 2015; 125:42-46.

6. Rao S, Tortola L, Perlot T, Wirnsberger G, Novatchkova M, Nitsch R, Sykacek P, Frank L, Schramek D, Komnenovic V, Sigl V, Aumayr K, Schmauss G, Fellner N, Handschuh $\mathrm{S}$, Glosmann M, et al. A dual role for autophagy in a murine model of lung cancer. Nat Commun. 2014; 5:3056.

7. Strohecker AM and White E. Autophagy promotes BrafV600E-driven lung tumorigenesis by preserving mitochondrial metabolism. Autophagy. 2014; 10:384-385.

8. Chen HY and White E. Role of autophagy in cancer prevention. Cancer Prev Res (Phila). 2011; 4:973-983.

9. Takamura A, Komatsu M, Hara T, Sakamoto A, Kishi C, Waguri S, Eishi Y, Hino O, Tanaka K and Mizushima N. Autophagy-deficient mice develop multiple liver tumors. Genes Dev. 2011; 25:795-800.

10. Parzych KR and Klionsky DJ. An overview of autophagy: morphology, mechanism, and regulation. Antioxid Redox Signal. 2014; 20:460-473.

11. Noda T, Matsunaga K, Taguchi-Atarashi N and Yoshimori T. Regulation of membrane biogenesis in autophagy via PI3P dynamics. Semin Cell Dev Biol. 2010; 21:671-676.

12. Nakatogawa H, Suzuki K, Kamada Y and Ohsumi Y. Dynamics and diversity in autophagy mechanisms: lessons from yeast. Nat Rev Mol Cell Biol. 2009; 10:458-467.

13. Shpilka T, Weidberg H, Pietrokovski S and Elazar Z. Atg8: an autophagy-related ubiquitin-like protein family. Genome Biol. 2011; 12:226.

14. Kabeya Y, Mizushima N, Ueno T, Yamamoto A, Kirisako T, Noda T, Kominami E, Ohsumi Y and Yoshimori T. LC3, a mammalian homologue of yeast Apg8p, is localized in autophagosome membranes after processing. EMBO J. 2000; 19:5720-5728.

15. Klionsky DJ, Abdelmohsen $\mathrm{K}$, Abe A, Abedin MJ, Abeliovich H, Acevedo Arozena A, Adachi H, Adams CM, Adams PD, Adeli K, Adhihetty PJ, Adler SG, Agam G, Agarwal R, Aghi MK, Agnello M, et al. Guidelines for the use and interpretation of assays for monitoring autophagy (3rd edition). Autophagy. 2016; 12:1-222.

16. Galluzzi L, Pietrocola F, Bravo-San Pedro JM, Amaravadi RK, Baehrecke EH, Cecconi F, Codogno P, Debnath J, Gewirtz DA, Karantza V, Kimmelman A, Kumar S, Levine B, Maiuri MC, Martin SJ, Penninger J, et al. Autophagy in malignant transformation and cancer progression. EMBO J. 2015; 34:856-880.

17. Mijaljica D, Nazarko TY, Brumell JH, Huang WP, Komatsu M, Prescott M, Simonsen A, Yamamoto A, Zhang H, Klionsky DJ and Devenish RJ. Receptor protein complexes are in control of autophagy. Autophagy. 2012; 8:1701-1705.

18. Rogov V, Dotsch V, Johansen T and Kirkin V. Interactions between autophagy receptors and ubiquitin-like proteins form the molecular basis for selective autophagy. Mol Cell. 2014; 53:167-178.

19. Inoue D, Suzuki T, Mitsuishi Y, Miki Y, Suzuki S,
Sugawara S, Watanabe M, Sakurada A, Endo C, Uruno A, Sasano H, Nakagawa T, Satoh K, Tanaka N, Kubo $\mathrm{H}$, Motohashi $\mathrm{H}$, et al. Accumulation of p62/SQSTM1 is associated with poor prognosis in patients with lung adenocarcinoma. Cancer Sci. 2012; 103:760-766.

20. Karpathiou G, Sivridis E, Koukourakis MI, Mikroulis D, Bouros D, Froudarakis ME and Giatromanolaki A. Lightchain $3 \mathrm{~A}$ autophagic activity and prognostic significance in non-small cell lung carcinomas. Chest. 2011; 140:127-134.

21. Zois CE, Giatromanolaki A, Sivridis E, Papaiakovou M, Kainulainen H and Koukourakis MI. «Autophagic flux» in normal mouse tissues: focus on endogenous LC3A processing. Autophagy. 2011; 7:1371-1378.

22. Martinet W, Schrijvers DM, Timmermans JP, Bult H and De Meyer GR. Immunohistochemical analysis of macroautophagy: recommendations and limitations. Autophagy. 2013; 9:386-402.

23. Mizushima $\mathrm{N}$ and Yoshimori $\mathrm{T}$. How to interpret LC3 immunoblotting. Autophagy. 2007; 3:542-545.

24. Schlafli AM, Berezowska S, Adams O, Langer R and Tschan MP. Reliable LC3 and p62 autophagy marker detection in formalin fixed paraffin embedded human tissue by immunohistochemistry. Eur J Histochem. 2015; 59:2481.

25. Liu JL, Chen FF, Lung J, Lo CH, Lee FH, Lu YC and Hung $\mathrm{CH}$. Prognostic significance of p62/SQSTM1 subcellular localization and LC3B in oral squamous cell carcinoma. Br J Cancer. 2014; 111:944-954.

26. Iwadate $\mathrm{R}$, Inoue J, Tsuda $\mathrm{H}$, Takano M, Furuya K, Hirasawa A, Aoki D and Inazawa J. High Expression of p62 Protein Is Associated with Poor Prognosis and Aggressive Phenotypes in Endometrial Cancer. The American journal of pathology. 2015; 185:2523-2533.

27. Luo RZ, Yuan ZY, Li M, Xi SY, Fu J and He J. Accumulation of $\mathrm{p} 62$ is associated with poor prognosis in patients with triple-negative breast cancer. Onco Targets Ther. 2013; 6:883-888.

28. Rolland P, Madjd Z, Durrant L, Ellis IO, Layfield R and Spendlove I. The ubiquitin-binding protein p62 is expressed in breast cancers showing features of aggressive disease. Endocr Relat Cancer. 2007; 14:73-80.

29. Burdelski C, Reiswich V, Hube-Magg C, Kluth M, Minner S, Koop C, Graefen M, Heinzer H, Tsourlakis MC, Wittmer C, Huland H, Simon R, Schlomm T, Sauter G and Steurer S. Cytoplasmic Accumulation of Sequestosome 1 (p62) Is a Predictor of Biochemical Recurrence, Rapid Tumor Cell Proliferation, and Genomic Instability in Prostate Cancer. Clin Cancer Res. 2015; 21:3471-3479.

30. Jiang T, Harder B, Rojo de la Vega M, Wong PK, Chapman E and Zhang DD. p62 links autophagy and Nrf2 signaling. Free Radic Biol Med. 2015; 88:199-204.

31. Inami Y, Waguri S, Sakamoto A, Kouno T, Nakada K, Hino O, Watanabe S, Ando J, Iwadate M, Yamamoto M, Lee MS, Tanaka K and Komatsu M. Persistent activation of Nrf2 
through p62 in hepatocellular carcinoma cells. J Cell Biol. 2011; 193:275-284.

32. Moscat J, Diaz-Meco MT and Wooten MW. Signal integration and diversification through the p62 scaffold protein. Trends Biochem Sci. 2007; 32:95-100.

33. Nakamura K, Kimple AJ, Siderovski DP and Johnson GL. PB1 domain interaction of p62/sequestosome 1 and MEKK3 regulates NF-kappaB activation. J Biol Chem. 2010; 285:2077-2089.

34. Pankiv S, Lamark T, Bruun JA, Overvatn A, Bjorkoy G and Johansen T. Nucleocytoplasmic shuttling of p62/SQSTM1 and its role in recruitment of nuclear polyubiquitinated proteins to promyelocytic leukemia bodies. J Biol Chem. 2010; 285:5941-5953.

35. Clausen TH, Lamark T, Isakson P, Finley K, Larsen KB, Brech A, Overvatn A, Stenmark H, Bjorkoy G, Simonsen A and Johansen T. p62/SQSTM1 and ALFY interact to facilitate the formation of p62 bodies/ALIS and their degradation by autophagy. Autophagy. 2010; 6:330-344.
36. Kau TR, Way JC and Silver PA. Nuclear transport and cancer: from mechanism to intervention. Nat Rev Cancer. 2004; 4:106-117.

37. Sobin LH, Gospodarowicz MK and Christian Wittekind C. (2009). International Union Against Cancer (UICC): TNM Classification of Malignant Tumours. Wiley-Blackwell), pp. 336.

38. Cihoric N, Savic S, Schneider S, Ackermann I, BichselNaef M, Schmid RA, Lardinois D, Gugger M, Bubendorf L, Zlobec I and Tapia C. Prognostic role of FGFR1 amplification in early-stage non-small cell lung cancer. Br J Cancer. 2014; 110:2914-2922.

39. Zlobec I, Suter G, Perren A and Lugli A. A next-generation tissue microarray (ngTMA) protocol for biomarker studies. J Vis Exp. 2014; :51893. 